OPEN ACCESS

Edited by:

Nicola Lettieri,

Istituto Nazionale per l'Analisi delle

Politiche Pubbliche (INAPP), Italy

Reviewed by:

Christopher R. von Rueden, University of Richmond, United States

Sam Sloss,

Indiana University Southeast,

United States

*Correspondence:

Judith M. Burkart

judith.burkart@aim.uzh.ch

Specialty section: This article was submitted to

Evolutionary Sociology and Biosociology,

a section of the journal

Frontiers in Sociology

Received: 06 November 2017

Accepted: 12 June 2018

Published: 09 July 2018

Citation:

Burkart JM, Brügger RK and van Schaik CP (2018) Evolutionary Origins of Morality: Insights From Non-human

Primates. Front. Sociol. 3:17.

doi: 10.3389/fsoc. 2018.00017

\section{Evolutionary Origins of Morality: Insights From Non-human Primates}

\author{
Judith M. Burkart*, Rahel K. Brügger and Carel P. van Schaik
}

Department of Anthropology, University of Zürich, Zurich, Switzerland

The aim of this contribution is to explore the origins of moral behavior and its underlying moral preferences and intuitions from an evolutionary perspective. Such a perspective encompasses both the ultimate, adaptive function of morality in our own species, as well as the phylogenetic distribution of morality and its key elements across primates. First, with regard to the ultimate function, we argue that human moral preferences are best construed as adaptations to the affordances of the fundamentally interdependent hunter-gatherer lifestyle of our hominin ancestors. Second, with regard to the phylogenetic origin, we show that even though full-blown human morality is unique to humans, several of its key elements are not. Furthermore, a review of evidence from non-human primates regarding prosocial concern, conformity, and the potential presence of universal, biologically anchored and arbitrary cultural norms shows that these elements of morality are not distributed evenly across primate species. This suggests that they have evolved along separate evolutionary trajectories. In particular, the element of prosocial concern most likely evolved in the context of shared infant care, which can be found in humans and some New World monkeys. Strikingly, many if not all of the elements of morality found in non-human primates are only evident in individualistic or dyadic contexts, but not as third-party reactions by truly uninvolved bystanders. We discuss several potential explanations for the unique presence of a systematic third-party perspective in humans, but focus particularly on mentalizing ability and language. Whereas both play an important role in present day, full-blown human morality, it appears unlikely that they played a causal role for the original emergence of morality. Rather, we suggest that the most plausible scenario to date is that human morality emerged because our hominin ancestors, equipped on the one hand with large and powerful brains inherited from their ape-like ancestor, and on the other hand with strong prosocial concern as a result of cooperative breeding, could evolve into an ever more interdependent social niche.

Keywords: evolution, morality, hunter-gatherers, prosociality, norm violations, concern for reputation, cooperative breeding, non-human primates

\section{INTRODUCTION}

Contemplation of law as a natural social phenomenon quickly reveals that it cannot be reduced to purely rational processes and explicit reasoning. It is fundamentally built on (albeit not identical with) our sense for morality, the propensity to differentiate actions, decisions, and intentions between those that are proper and right and those that are improper or wrong 
(Long and Sedley, 1987). This evaluation can be the result of deliberation, but also of automatic proximate mechanisms such as intuitions that are expressed by a variety of moral emotions, motivations, and preferences which often have a high-urgency feel (Weaver et al., 2014).

Social scientists have traditionally considered morality as a recent, purely cultural innovation, seemingly necessary to keep our otherwise brutish nature under control (e.g., reviewed in Long and Sedley, 1987; de Waal, 2006; Haidt, 2013). In support of this conjecture, what is considered moral in a given culture or society, or what the corresponding systems of laws prescribe, can indeed be quite variable. However, despite this variability in the content of what counts as moral among cultures, there are also elements that seem universal, both with regard to the proximate mechanisms that regulate moral behavior and the content of moral norms. For instance, Barrett et al. (2016) found that across societies, including small-scale societies, humans take an agent's reason for action into account for moral judgments, but they also found independent variation when looking at specific contents, e.g., harm vs. theft, or in how the content influences the role of intentionality. Furthermore, even if conformist transmission could in principle stabilize a variety of behaviors and norms (Chudek and Henrich, 2011), there appears strong canalization in that some kinds of content (such as for instance not to harm others, or engage in parental investment) are more readily considered moral than others (van Schaik, 2016).

Ubiquitous key elements of human morality discussed in this paper are prosocial concern and conformity, as well as the moral contents of doing good, not harming others, and avoiding inequity and incest (van Schaik, 2016). Importantly, these elements are not only expressed when the individual is personally involved, i.e., in individualistic or dyadic contexts, but also in the absence of personal involvement, i.e., in third-party contexts. For instance, moral behavior not only includes the urge to conform to the rules and norms of one's own community, but also evokes strong feelings that others ought to do so as well. The universal presence of these elements of morality across human societies suggests there is an evolved core to morality, which should therefore be amenable to a functional and comparative evolutionary analysis sensu Tinbergen (Tinbergen, 1963; Bateson and Laland, 2013).

Such an evolutionary analysis claims that whenever universal, proximate mechanisms have evolved, they must have done so to fulfill a specific adaptive function. In the first section of this contribution we will argue that the adaptive function of our evolved morality was to enable the highly interdependent life-style of Pleistocene hunter-gathers.

An evolutionary analysis of human morality also includes the examination of its phylogenetic origin, to which we will turn in the second section. Whereas full-blown human morality, which includes explicit moral reasoning and evaluation, may well be unique to humans, some of its elements or building blocks are not, and we can use data from non-human primates to trace the evolutionary history of each of them separately. An obvious first, and very popular, step is to look at the great apes, and in particular the chimpanzees and bonobos (e.g., de Waal, 2006), to investigate the possible presence of a specific building block in our closest relatives. However, a broader and more informative comparative approach consists in mapping the presence or absence of each of these building blocks or traits in a broader set of species, to then test which factor best predicts this pattern of distribution (MacLean, 2016). If the specific case of humans fits such an identified pattern, this allows us to identify the evolutionary context of the emergence of this trait. This approach thus ideally allows not only to identify that a trait is or is not unique to humans, but also why it is present in a given set of species, including humans.

\section{HUNTER-GATHERERS: THE EVOLUTIONARY CONTEXT OF THE EMERGENCE OF HUMAN MORALITY}

As a species, humans have spent $95 \%$ of their evolutionary past as Pleistocene hunter-gatherers (Hill et al., 2011). Even though we cannot travel back in time and observe how these people lived, the few remaining hunter-gatherer societies across the globe allow us a glimpse into our evolutionary past, by providing useful models for the reconstruction of ancestral selection pressures. Intriguingly, despite often considerable geographical distance and principled variation, these societies are rather homogeneous (Marlowe, 2005), and the communalities between them therefore are likely representative for the evolutionary context in which human sociality in general, and thus morality, has evolved.

Nomadic hunter-gatherers live in highly interdependent, egalitarian societies (Marlowe, 2005). Even though some individuals can be more influential than others, major decisions are usually made collectively. In fact, if some individual tries to rise to a leader position through coercive leadership in order to dominate the rest of the group, the majority will try to prevent this (Boehm, 2012). Hunter-gatherers form socially recognized pair bonds (i.e., marriages), and show a marked sexual division of labor: women gather and men hunt cooperatively, fish, or collect honey (Marlowe, 2007). The foraging niche is skill-intensive and often requires intense cooperation. The skills are socially transmitted and shaped by cumulative cultural evolution (Dean et al., 2014; Hill et al., 2014), and it takes women until their midtwenties, and men even longer, to become fully efficient foragers. The social structure and networks of hunter-gatherers in fact appears to optimize efficient transmission of cultural knowledge. Either sex may disperse, but adult brothers and sisters often coreside. Most individuals in the group are unrelated, and strong ties with non-kin play an important role for the spread of skills and knowledge (Hill et al., 2011; Migliano et al., 2017).

Hunter-gatherer lives are characterized by high levels of interdependence in almost all contexts and at different timescales. Food sharing is vital, at the time scale of days (hunters, but also foragers, may return empty-handed), weeks to months (in case of sickness or injury), and years to decades (families with growing children do not produce enough and rely on younger and older camp members: Sugiyama and Chacon, 2005; Kaplan et al., 2009; Hill et al., 2011). Gathered food is generally shared within families, but honey and meat, in particular from large animals, which are hunted cooperatively, are shared with 
all other families in a camp (Wood and Marlowe, 2013). In general, food is shared with those who are needy, but also preferentially with those who have shared in the past. It is thus crucial that someone build a good reputation and support others without being solicited, to ensure receiving support when needy themselves. A good reputation is thus vital, because sooner or later this need will arrive. Men can gain status by being generous (Gurven et al., 2000; Marlowe, 2010), and by participating in coordinated collective action, as during warfare, cooperative hunting, gathering, or moving camp.

Not only subsistence and foraging activities are fundamentally cooperative, but also child rearing. For a mother, it is almost impossible to rear a child successfully by herself, and she receives ample support from others, in particular fathers, grandmothers and older siblings, but also from other camp members (Hrdy, 2009). In fact, humans qualify as cooperative breeders, a reproductive system also known in several other animals, such as many bird species, but also wolves, or callitrichid monkeys. In all these species, including humans (Sear and Mace, 2008), parents obtain a significant amount of help in rearing their offspring, and both growth and survival of the offspring depends on the availability of helpers. Cooperative breeding typically evolves when conditions are harsh, which makes it increasingly difficult for mothers to raise their offspring alone (Burkart et al., 2017b). When our hominin ancestors moved into the savanna, food was more often dispersed and hidden underground than before. This required not only more cooperation during foraging and more elaborate food processing techniques (e.g., cooking: Wrangham, 2009), but also made it more difficult for mothers to rear their offspring independently. This is in strong contrast to all the other great apes, where mothers raise their offspring independently and are well able to do so. Furthermore, large brains require large amounts of energy, in particular during ontogeny (Kuzawa et al., 2014). It is thus parsimonious to assume that our ancestors had already started to engage in systematic allomaternal care rather early since otherwise the evolution of our big brains would not have been possible (Isler and van Schaik, 2012).

Human morality can be understood as a straight-forward adaptation to this hunter-gatherer life-style, in that it enables and stabilizes interdependence (see also van Schaik et al., 2014). According to this hypothesis, one key element of morality, a prosocial predisposition, is crucial to maintain food sharing with immatures and adults. Having a good reputation serves as insurance to being cared for when in need, and also for being chosen as a mate or cooperation partner. A strong concern for one's reputation, including reputation management, thus ensues. The second element, an urge to conform, is crucial in a niche where coordinated or synchronized action is vital for survival. In addition, the urge to conform serves to acquire the many complex skills that make up our ecological niche via social learning. When skills and knowledge are opaque, i.e., when it is not obvious how separate steps involved in an activity lead to an overall goal, trustful copying even of seemingly useless elements is mandatory (Henrich and Broesch, 2011; Dean et al., 2014).

The ultimate function of human morality and its key elements can thus readily be understood as an adaptation to the huntergatherer lifestyle. But are these elements unique to humans, or can some of them, or perhaps their precursors, also be found in other primates, and if so, why? These questions are important because a better understanding of the phylogenetic origins of elements of morality in non-human species can help evaluate the functional hypothesis that human morality has evolved to solve problems inherent to a fundamentally interdependent lifestyle.

\section{EVOLUTIONARY ORIGINS OF THE BUILDING BLOCKS OF MORALITY}

Building blocks of morality include both mechanisms and contents. For clarity, we discuss them separately (mechanisms: section Prosocial Concern, and section Conformity; contents: section Social Norms I: Universal, Biologically Anchored Contents, and section Social Norms II: Arbitrary, Culturally Variable Norms). However, links between them exist and will be addressed in the corresponding sections. An important issue for full-blown morality that applies to all building blocks is whether they are expressed in individualistic or dyadic contexts only, or whether they are also present in third-party contexts. For instance, can a prosocial concern in a given species be found between an actor and a recipient only, or do non-involved third parties (i.e., non-involved bystanders) also evaluate the prosocial interaction between an actor and a recipient as morally appropriate? This third-party perspective is an overarching hallmark of human morality in general and we will therefore also focus on this particular aspect when reviewing the evidence from non-human animals.

\section{Prosocial Concern}

One key element of human morality is prosocial concern, i.e., a concern not only with one's own but also with others' well-being, also referred to as other-regarding preferences by behavioral economists (Fehr and Fischbacher, 2003). In the primatological literature, it is often referred to as proactive prosociality, to stress that the corresponding behaviors, such as for instance food sharing, are not the result of solicitation by recipients, begging, or even harassment, but that they are initiated spontaneously by the actor without triggering by other individuals (Jaeggi et al., 2010).

Over the last decade, proactive prosociality has been extensively studied in a number of primate species. Early studies found it was absent in chimpanzees, who are independent breeders, but present in the small marmoset monkeys, who like humans, are cooperative breeders (Cronin, 2012; MarshallPescini et al., 2016). Importantly, even though the evolution of cooperative breeding is based on inclusive fitness benefits (Burkart et al., 2017b), kin selection and relatedness per se cannot explain why some primates show proactive prosociality but others don't. First, marmosets can show proactive prosociality toward non-related group members as well, and even strangers who are potential group members (Burkart et al., 2007). Second, highly related mother-offspring dyads in independently breeding primates, including chimpanzees (Ueno and Matsuzawa, 2004), fail to show proactive prosociality.

Later prosociality studies produced more mixed results, also because different methodologies make it difficult to compare 
between studies and species (Burkart and Rueth, 2013). A large comparative study therefore compared proactive prosociality across 15 primate species, using exactly the same methodology and thus providing directly comparable data. Phylogenetic analyses revealed that the extent of allomaternal care, (i.e., the amount of help that mothers receive from others when rearing infants, with cooperative breeding found in the higher range of values) is indeed the best predictor for proactive prosociality in a group service paradigm, whereas brain size or other socioecological factors cannot explain a significant amount of interspecific variation (Burkart et al., 2014).

Accordingly, chimpanzees, our closest relatives, scored low on prosociality. Nevertheless, their score was not zero, which corresponds to reports of occasional targeted helping in this species (Warneken and Tomasello, 2015; but see Tennie et al., 2016), as well as occasional food sharing or alerting others of danger. In addition to prosociality, targeted helping also has an important cognitive component, which is particularly strong in the large-brained apes (Burkart et al., 2017a). A highly relevant test case are bonobos, for which evidence for proactive prosociality is quite mixed (Tan and Hare, 2013; Tan et al., 2015 , 2017), but who unfortunately were not in the cross-species sample of the group service study.

In sum, among primates, proactive prosociality increases with the amount of allomaternal care found in a species and culminates in cooperative breeders. Since humans also qualify as cooperative breeders, it is most parsimonious to conclude that our prosociality is simply the result of cooperative breeding too, i.e., that the same regularity applies to non-human and human primates alike (Burkart et al., 2014).

So far, primate proactive prosociality has mostly been studied from the dyadic perspective. However, in humans, it also encompasses the third-party context. Social evaluation studies address whether subjects, after observing how target individuals interact with others, avoid antisocial target individuals (and thus show a negativity bias) or prefer prosocial and cooperative target individuals (positivity bias). For instance, babies already have a preference for agents who help, rather than hinder others (Hamlin et al., 2007). Such studies are also increasingly done with non-human animals, as reviewed in Abdai and Miklósi (2016). For instance, in a study modeled after Hamlin et al. (2007), bonobos unexpectedly showed a preference for hinderers, rather than helpers (Krupenye and Hare, 2018). Abdai and Miklósi (2016) point out that there are still considerable conceptual and procedural issues in animal social evaluation studies, in particular to clearly demonstrate positivity biases. Negativity biases may be taxonomically far more widespread than positivity biases, since the need to avoid harm is universal whereas the need to cooperate is less common. Evidence for positivity biases (which correspond to the third-party perspective on prosociality) appears present too in several non-human primate species but is more elusive due to methodological issues, including the use of humans rather than conspecifics as target individuals (see Abdai and Miklósi, 2016).

An important aspect of human prosociality directly follows from the fact that we evaluate people based on their prosocial behavior toward others. When deciding whether to behave prosocially or not, we are highly sensitive to a potential audience. We thus strongly care not only about to what extent others behave prosocially, but also about whether others perceive $u s$ as prosocial and thus reliable partners (Goffman, 1959). In dictator games, which are used by behavioral economists to quantify other-regarding preferences, humans typically contribute a nonzero amount of money even if they could keep this money for themselves without any negative consequences, consistent with proactive other-regarding preferences (Fehr and Fischbacher, 2003). However, when the same game is played and stylized eye-cues are added on the answer sheet, these "watching eyes" elicit increased prosocial donations in such games, which reflects our strong concern for reputation (Nettle et al., 2013b). In corresponding experiments with chimpanzees, the same effect was not found, and the authors concluded that the extreme human sensitivity to cues of potential conspecific observation appears absent in chimpanzees (Nettle et al., 2013a, see also Engelmann et al., 2012).

These findings suggest that chimpanzees are perhaps not the best species to look for such effects. Rather, these effects would arguably be most likely in habitually prosocial species, such as the cooperatively breeding marmoset monkeys. We therefore studied audience effects on prosocial behavior in this species in a naturalistic context, i.e., proactive food sharing with immatures (Brügger et al., 2018). Marmosets live in family groups, and all members contribute to infant rearing. When the infants are small, they are carried by all group members, and in big and wellestablished groups they sometimes are only handed back to the mother for breastfeeding. When the infants are older and ingest solid food, all group members share food with the immatures. This food sharing can take the form of proactive food sharing, i.e., food is offered to the immatures without previous begging, even when immatures are not even aware that a valuable food item has been found. To test for an audience effect on proactive food sharing, we quantified food sharing by helpers with immatures, either when they were alone with the offspring in a separate room or when the rest of the family was present. If they were sharing food to increase their reputation of being a good helper, one would expect them to share more when an audience was present than when they were alone with the offspring. The marmosets were sensitive to the audience, but in the opposite direction than expected: they showed more proactive food sharing in the absence of an audience. This effect is in fact consistent with the well-established bystander apathy (Latané and Darley, 1969) or diffusion of responsibility (Bierhoff, 2017) effect in humans. Thus, the marmosets perhaps shared more because they felt more "responsible" to fulfill the immatures' needs when no one else was around, but in any case, these results show that they did not take advantage of this situation to engage in reputation management.

To summarize, a genuine proactive prosocial concern is not unique to humans but we also see it in other primates, in particular in those who like humans engage in cooperative breeding. Nevertheless, to date there is no solid evidence that primates would take into account whether others behave prosocially or not. Thus, a third-party perspective on prosociality appears largely lacking in primates.

\section{Conformity}

A second key element of human morality is conformity. Conformity can be conceptualized in different ways (Van 
Leeuwen et al., 2015; Whiten and van de Waal, 2016a), from copying the majority, to copying a new behavioral variant while abandoning a personal preference for a previously acquired behavioral variant, to not only copying the majority but doing so with a disproportionate probability. Empirical evidence for such a disproportionate tendency is scarce in humans (Acerbi et al., 2016) and also among animals (Aplin et al., 2017). However, there is increasing evidence in primates for the other forms of conformity.

Even though chimpanzees may sometimes be reluctant to give up their personal preferences for learned behaviors (Hrubesch et al., 2009), other studies have shown that a specific foraging technique seeded in a group will spread within this group. Intriguingly, even individuals who independently discovered an alternative solution would tend to stick to the seeded solution that was most prevalent in the group (Whiten et al., 2005, 2007). A similar pattern was also found for capuchin monkeys (Dindo et al., 2009). Thus, depending on the specific context, primates can be either conservative (i.e., stick to their personal solution) or conformist (Hopper et al., 2011).

Perhaps the most striking evidence for conformity in nonhuman primates comes from vervet monkeys. Immigrating males, who in their origin group developed a strong preference for one type of novel food (artificially colored blue or pink maize of identical taste), immediately changed their preference after immigrating in a group where the majority of individuals preferred the other color (van de Waal et al., 2013; Whiten and van de Waal, 2016a). The function of this kind of informational conformity is most likely to quickly adapt to the local knowledge of the new group. Strong informational conformity also enables naïve individuals to socially learn cognitively opaque skills where the causal role of each single step in achieving the overall goal is not obvious. Thus, a preference for social knowledge over private knowledge, or an urge to conform can be understood in the service of self-interest, i.e., to acquire reliable information about the world.

An interesting phenomenon, which questions whether all primate conformity is informational, has recently been reported from the same vervet monkey population. In one of the study groups, a small number of subordinate females split off to form its own group. In the original parent group, the individuals preferred blue maize. However, since the females from the future splinter group were subordinate, they had not always had access to the preferred food, and therefore also had repeatedly sampled the pink maize, with some having eaten even more pink than blue maize in the parent group. After group fission, all these previously subordinate females could now freely express their preference in the new splinter group, and continued to show a strong preference for blue maize (even after 4 month, they ate $100 \%$ of the time blue maize). They thus still conformed to the preference of the parent group even though they were no longer surrounded by individuals from the parent group, and even though there was no difference in the taste between the blue and the pink maize (in fact, some never even knew that one or the other color had been unpalatable). There can thus be remarkable resilience in preferences established through social learning, at least in vervet monkeys, and the authors propose the notion of social conformity, i.e., that individuals act like others to achieve a social function and simply be "like others", rather than to achieve an informational benefit (van de Waal et al., 2017). In fact, being more similar to others may facilitate group integration, and increasing evidence suggests that primates indeed prefer others who are more similar to themselves as social partners (Paukner et al., 2009; Massen and Koski, 2014; Capitanio et al., 2017; Ruch et al., 2018).

In sum, at the individual level, several instances of conformity can be found in nonhuman primates, which can result in informational and perhaps also social benefits. But what about the third-party perspective? The third-party perspective becomes obvious in normative conformity, which consists not only of an individual's urge to conform (the individual perspective), but also of the expectation of others that the individual converges to their group's norms (the third party perspective). In general, as we will review below, normative conformity is absent in non-human primates, but the situation may be slightly more differentiated, depending on the specific norm that is at stake. In the following sections, we therefore turn to specific contents of potential norms in primates, making the fundamental distinction between (putative) social norms that have a universal and presumably biologically anchored vs. an arbitrary and culturally variable content.

\section{Social Norms I: Universal, Biologically Anchored Contents}

The contents of morality are norms, which permeate every aspect of human life and systematically guide our behavior (Gelfand and Jackson, 2016). Social norms are notoriously difficult to define. They broadly refer to implicit or explicit rules that prescribe behavior, whose violation elicits social sanctions. They range from being more or less compulsory (e.g., not to murder vs. to eat with chop-sticks), to being just common sense, as in conventions. Their content can be arbitrary and therefore culturally variable, or universal and biologically anchored. The two distinctions often overlap, but not always. For instance, not to commit murder is most likely compulsory and universal in most societies, but also more arbitrary norms such as taking off one's shoes before entering a house can be compulsory in a given society. Universal norms, however, tend to be compulsory in most societies (for a more detailed discussion of the nature of norms, see also Rudolf von Rohr et al., 2011).

Identifying universal moral norms in humans is still an ongoing endeavor (e.g., Barrett et al., 2016) but they minimally include the contents of not harming infants, avoiding inequity, caring for one's own offspring, and avoiding incest. Some evidence suggests that they may be present at least in dyadic, but sometimes even in third-party contexts in non-human primates too. In dyadic contexts, they are observed when interaction partners expect each other to behave in a specific way consistent with a potential norm, whereas in third-party contexts, uninvolved bystanders who observe interactions between other dyads would expect these dyad partners to interact in this way, and experience disapproval or even show moralistic aggression upon violations of this expectation. 
Among chimpanzees, for instance (but also among many other primates), infants usually enjoy high levels of tolerance and are hardly ever harmed by others. However, exceptions exist, which eventually even include infanticide (Townsend et al., 2007). Naturalistic observations show that bystanders who observe harmful behaviors often show strong reactions (Goodall, 1971; de Waal, 1991), such as waa barks (protest vocalizations: Clay et al., 2016), and even direct interventions in the form of policing (Rudolf von Rohr et al., 2012). Harmful behaviors toward infants elicit particularly strong reactions (Goodall, 1977; Townsend et al., 2007), such as interventions and defense of the mother-infant pair by multiple group members, sometimes culminating in highly dramatic situations. These behaviors are consistent with a strong third-party bystander reaction toward infanticide. However, it is important to note that these bystanders are not completely uninvolved, since they (e.g., other mothers with dependent offspring) may still have a very strong individual stake in discouraging infanticide by group males. We therefore presented captive chimpanzees with video clips of infanticide committed by completely unaffected third parties (conspecifics in the wild). The chimpanzees indeed clearly reacted to this norm violation: they paid far more attention to these clips compared with control videos depicting hunting scenes (lethal aggression against small hetero-specific monkeys), grooming and nut cracking, or displays and aggression between adult chimpanzees. However, this expectation violation as evident in looking times did not also translate in higher levels of arousal. Together, these results suggest that chimpanzees do indeed react strongly toward the violation of the putative norm "do not harm infants," including indignation-like expressions, but only if this happens in the within-group context. As truly non-involved bystanders, they still appear to detect such a putative norm violation, but this is not accompanied by overt disapproval (e.g., no arousal, and no waa-barks; Rudolf von Rohr et al., 2015). It is worth pointing out that in humans too, morality tends to be parochial in that we feel stronger about norm violations within the group (Fessler et al., 2015, but see Piazza and Sousa, 2016), and that punishment of norm violations is often not altruisitic (Guala, 2012).

Vervet monkey mothers too appear to expect that others don't harm their infants, and adult males behave accordingly (Hector et al., 1989). In an experiment, vervet males showed less aggression toward an infant if the infant's mother could see them compared to when not. Furthermore, mothers were more aggressive toward males after separation when they had observed the male behaving aggressively to the infant through a oneway mirror. Thus, vervet mothers appear to evaluate the males based on their behavior toward infants (and can act accordingly, because of minor sexual size dimorphism), and males adjust their behavior toward infants depending on whether they could be seen by the mothers. But again, mothers don't count as truly non-involved bystanders because they have high stakes in the well-being of their infants.

Inequity aversion is another content particularly closely related to morality (Decety and Yoder, 2017). It can take the form of disadvantageous inequity aversion, i.e., an aversion against being treated unfairly, such as receiving a lower reward for the same amount of work compared to a partner, which is egocentric. Alternatively, it can be advantageous inequity aversion, i.e., an aversion against obtaining a reward that is higher than that of a partner, which is therefore allocentric. In humans, both forms can already be observed in 3 year old toddlers (Ulber et al., 2017). Inequity aversion has also been reported in a variety of primate species (reviewed in Talbot et al., 2016; Ulber et al., 2017; but see Engelmann et al., 2017), but only in the egocentric form (but see Brosnan et al., 2010).

Disadvantageous inequity aversion is consistent with an individualistic perspective on one's own benefit, whereas advantageous inequity aversion includes a prominent prosocial element and is a particularly strong indicator for a concern with equity per se. However, both types of inequity aversion include personal involvement, which can automatically trigger a self-serving bias that may overshadow equity preferences. This methodological problem can be overcome by quantifying inequity aversion in third-party contexts. In third-party contexts, the question is whether individuals have a preference for the fairness between third parties, and self-serving biases therefore no longer interfere. This can be a preference for a fair distribution of rewards (i.e., fifty-fifty in dyads), but also a preference for distributions that take into account merit and wealth, which has been shown to be already present in young children (Kanngiesser and Warneken, 2012; Paulus, 2014). We are not aware of any work on this among non-human primates.

A final universal and biologically determined content of human social norms is incest avoidance. Incest avoidance if often construed as a cultural taboo (Turner and Maryanski, 2015), and we have strong third-party attitudes toward it, for instance including indignation and disgust in the case of sibling sex (Fessler and Navarrete, 2004). Nevertheless, incest avoidance is also practiced by non-human primates (Bischof, 1975; Pusey and Wolf, 1996), and is particularly strong in callitrichid monkeys (marmosets and tamarins: Saltzman, 2003; Saltzman et al., 2004). For instance, opposite-sex callitrichid siblings can be kept for years without them engaging in reproduction or sexual behavior, and also fathers show no sexual interest in their reproductively mature daughters. These preferences are especially adaptive in the callitrichid social system because offspring of both sexes often remain in their natal group for extended periods of time and help raise their younger siblings. But again, unlike in humans (Fessler and Navarrete, 2004), there is no evidence that other group members or even non-involved third parties would object to close kin having sexual relationships (although we are not aware of any direct test of this idea).

Taken together, non-human primates often have clear expectations about how others should interact with them. In fact, we can readily add other examples, such as the expectation of dominants regarding how subordinates should behave toward them, or how a partner should behave during playful interactions. Thus, natural social rules appear ubiquitous in primates and in fact in other gregarious species too, but a crucial limitation in most cases is that these rules are applied only to actual or potential partners but not in third-party contexts when uninvolved bystanders are concerned. Exceptions may be found in some specific contexts, such as shown in the case of infanticide 
in chimpanzees, who show strong bystander reactions, as long as norm violations occur within their social group but not when they occur in complete stranger conspecifics. Similar reactions are not unlikely in other primate species susceptible to male infanticide (van Schaik and Janson, 2000).

\section{Social Norms II: Arbitrary, Culturally Variable Contents}

Arbitrary, culturally variable norms are particularly salient elements of human morality, and at least in part responsible for the traditional view that morality is a purely cultural innovation (de Waal, 2006; Haidt, 2013). Nevertheless, cultural behavioral variation has also been described for non-human primates and other animals (Whiten and van de Waal, 2016b). Behavioral innovations can spread via social learning within populations, which leads to cultural differences in behavioral repertoires, and these are particularly large in great ape species such as chimpanzees (Whiten et al., 2017) and orangutans (van Schaik et al., 2009). These cultures differ from human cultures in that they are not cumulative and also not symbolic (Gruber et al., 2015).

As discussed in detail in section Conformity, such primate cultures can be supported by strong informational and perhaps social conformity. Nevertheless, they are not supported by normative conformity. In other words, individuals may be eager to conform to other group members even in the case of arbitrary, culturally variable behaviors (e.g., eating pink instead of blue corn in vervet monekys: van de Waal et al., 2013, or specific tool use techniques in chimpanzees: Luncz and Boesch, 2014), but the group members who are performing the majority behavior appear to have no stake in whether others conform or not, let alone show signs of indignation or even punishment of nonconformers. This might also explain why conformity can also be absent in cultural behaviors, as for instance in high-arm grooming among chimpanzees (Wrangham et al., 2016).

\section{CONCLUSIONS}

Our goal was to provide an overview over the current state of the art on research into the evolutionary origin of morality (see also van Schaik et al., 2014). We did so by analyzing both its ultimate function in our own species, and investigating the phylogenetic origin of elements of human morality in non-human primates.

We propose that the ultimate function of human morality is best understood as a straightforward adaptation that enabled the fundamentally interdependent lifestyle of our hunter-gatherer ancestors. Even though full-blown morality is most likely unique to humans, several of its key elements can be found in nonhuman primates and some other animals. Our goal was to provide an overview of the occurrence of such elements in nonhuman primates, to understand under what conditions they emerged during evolution, and to better delineate in which ways human morality is unique (Figure 1). We therefore focused on two key components of morality, i.e., a prosocial concern and conformity, and the contents of natural and arbitrary, cultural norms. These elements are arguably necessary and crucial for the emergence of morality, but not sufficient. Additional elements include language (discussed below), parochialism (Baumgartner et al., 2012; Fessler et al., 2015), and perhaps coalitional psychology (DeScioli and Kurzban, 2013). For the elements discussed in this paper, there is evidence from non-human primates and sometimes from other animals as well. The contents can be highly species-specific, adapted to the social requirements of a given species. An evolutionary perspective on moral behavior therefore suggests that with regard to content, it may be useful to distinguish between human morality, chimpanzee morality, marmoset morality, and so on.

Intriguingly, some of these elements of morality are not necessarily most prevalent in our closest relatives, the great apes. In particular the key element of prosocial concern is stronger in primates that show more similarities in social structure with humans, namely the cooperatively breeding callitrichid monkeys. The lives of humans are very much different from that of other great apes, in modern societies as in hunter-gatherers. Compared to non-human great apes, every domain of our lives is built on high levels of interdependence (van Schaik and Burkart, 2010; Tomasello, 2016), from subsistence (foraging) and institutionalized activities to rearing children. Nevertheless, a high degree of interdependence is not unique to humans, but also present in other primates, in particular in cooperatively breeding callitrichid monkeys who also raise their offspring with the help of all group members (Hrdy, 2009). It thus appears that some of the elements of morality evolved convergently in highly cooperative, interdependent species (such as humans and callitrichid monkeys) but not in more individualistic ones (such as chimpanzees). Based on comparative studies, we have argued that interdependence during infant rearing is key, but others place greater emphasis on the role of interdependence during foraging (Tomasello et al., 2012; Tomasello and GonzalezCabrera, 2017). Note, however, that arguably, the latter could only emerge once some basic proactive prosociality that facilitates cooperation and sharing had evolved in the context of shared offspring care (van Schaik and Burkart, 2010).

Obviously, cooperatively breeding callitrichid monkeys don't have full-blown human morality, perhaps because more cognitively demanding elements of morality appear well-beyond the capacities of these small brained monkeys. Systematic comparative analyses confirm that cognitive abilities across non-human primates are correlated with brain size, and humans fit this pattern too (Burkart et al., 2017a). Accordingly, the very big brained great apes have many remarkable cognitive abilities, and a valid working hypothesis is that it was the unique coincidence in our ancestors of two elements that enabled fullblown morality: the strong cognitive abilities, supported by big brains and inherited from our common ancestors with the other great apes on the one hand, and on the other hand our strong prosocial concern, which was added convergently because our ancestors started to engage in cooperative infant care sometime after they had diverged from the other great ape lineage (Burkart et al., 2009; Burkart and van Schaik, 2016). For instance, great apes, but not callitrichids, appear to show disadvantageous inequity aversion in that they are upset when they receive a lower-value reward compared to a social partner (Talbot et al., 


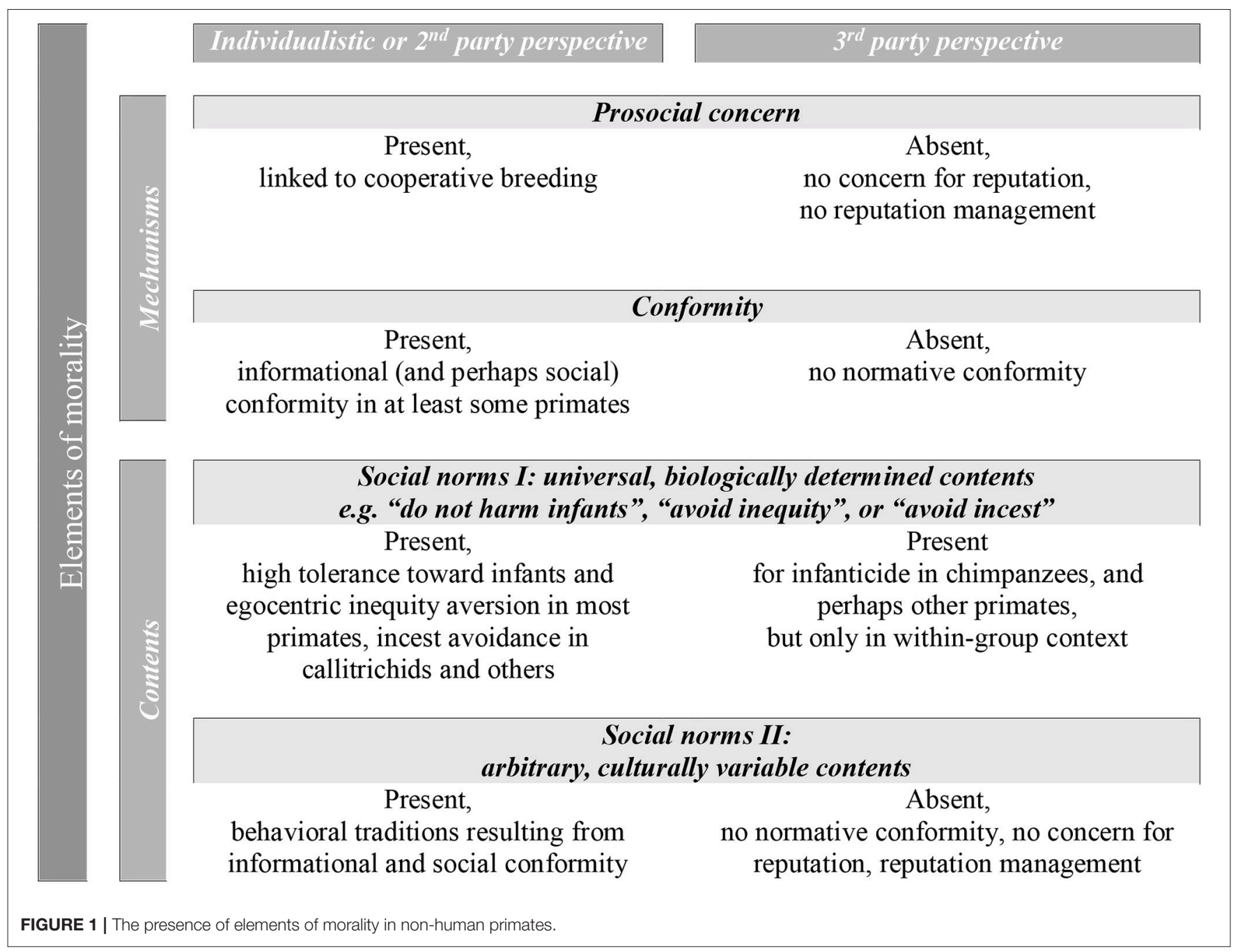

2016). This suggests that they are accurately keeping track of each other's efforts and the amount of reward obtained for it. However, only when this cognitive precondition is coupled with a stable prosocial concern for others (as it is the case in our own species only) may we also see disadvantageous inequity aversion, or an aversion from the perspective of an uninvolved bystander toward inequity between two social partners.

A striking pattern for all these elements is that even though they are present in at least some primate species, they are fundamentally restricted to the individual perspective in nonhuman primates (Figure 1). We can imagine three possible, non-exclusive explanations for the emergence of the third-party perspective in human morality. First, the third-party perspective may simply require even more sophisticated mentalizing abilities and perhaps language. However, a third-party perspective is not even taken systematically by chimpanzees, for whom increasing evidence for quite impressive mentalizing abilities is available (Krupenye et al., 2016). Likewise, the case of human psychopaths suggests that language per se is at least not a sufficient condition for full-blown human morality (Hare, 1999).
A second possible explanation for the origin of the thirdparty perspective, in particular in the case of conformity, emerges in the face of increasing evidence for assortative preferences in primates (see also Haun and Over, 2015 for a similar argument). This body of evidence suggests that primates have a general preference for partners that are like themselves (Paukner et al., 2009; Massen and Koski, 2014; Capitanio et al., 2017; Ruch et al., 2018). In situations of high interdependence at the group level, it is important for individuals to have reliable relationships with all group members, which can result in a preference for ego to conform to the behaviors of the group. However, it may also mean that the group members themselves now have a stake in newcomers to conform, i.e., normative expectations. In other words, similarity, for instance in vocal communication, may turn into a tag for affiliation and cooperation (Cohen et al., 2012; Ruch et al., 2018) and signal an individuals' readiness to be a reliable member of the group, which is important for all group members. For the individual, this will lead to an increasing urge to conform not only for informational, but also for social reasons (van de Waal et al., 2017). In other group members, it will lead to the normative expectation that a specific individual indeed 
conform. This explanation is also consistent with studies that find only weak evidence for the idea that group norms are the only or principal explanation for third-party moral judgments in humans (Krasnow et al., 2012; Delton and Krasnow, 2017), or that norm-based punishment is not necessarily altruistic (Guala, 2012).

Finally, the third-party perspective may simply have emerged as a byproduct of the increasing importance of indirect reciprocity, which is an essential element of the ecological interdependence of human foragers. Indirect reciprocity critically involves the observation and evaluation of interactions between third parties, so as to assess the suitability of each participant as recipient of prosocial actions and thus as a partner in exchanges. Once such evaluations of third parties are shared with others in the cooperation network through language, they can easily acquire the same emotional status as judgments about direct partners, i.e., second parties.

Language may thus have played a crucial role for the evolution of full-blown human morality. First, as just argued, it amplifies the importance of reputation effects, because misbehavior can be made public via gossip to the entire group. Language thus likely is the key driver for our obsession with reputation (see also van Schaik, 2016), and that even most subtle cues of being observed can significantly influence our behavior (Nettle et al., 2013b). Second, language enables negotiation and therefore the formation of formal, explicit and institutionalized rules, and thus can lead to culturally modified contents of social and moral norms. Linked to this, language and language-based moral reflection may well be responsible for the finding that the truly independent third-party perspective (i.e., also including out-group contexts) is only found in humans. Unreflected moral judgments, emotions, and preferences are typically highly parochial: humans spontaneously care most about their in-group, in particular when we are under duress (Baumgartner et al., 2012; De Dreu et al., 2015; Fessler et al., 2015). This in-group bias can be overcome when we manage to view out-group individuals at least as potential in-group members, but also via explicit, language-based moral reasoning.

\section{REFERENCES}

Abdai, J., and Miklósi, Á. (2016). The origin of social evaluation, social eavesdropping, reputation formation, image scoring or what you will. Front. Psychol. 7:1772. doi: 10.3389/fpsyg.2016.01772

Acerbi, A., Van Leeuwen, E. J., Haun, D. B., and Tennie, C. (2016). Conformity cannot be identified based on population-level signatures. Sci. Rep. 6:36068. doi: $10.1038 /$ srep36068

Aplin, L. M., Sheldon, B. C., and McElreath, R. (2017). Conformity does not perpetuate suboptimal traditions in a wild population of songbirds. Proc. Natl. Acad. Sci. U.S.A. 114, 7830-7837. doi: 10.1073/pnas.1621067114

Barrett, H. C., Bolyanatz, A., Crittenden, A.N., Fessler, D. M., Fitzpatrick, S., Gurven, M., et al. (2016). Small-scale societies exhibit fundamental variation in the role of intentions in moral judgment. Proc. Natl. Acad. Sci. U.S.A. 113, 4688-4693. doi: 10.1073/pnas.1522070113

Bateson, P., and Laland, K. N. (2013). Tinbergen's four questions: an appreciation and an update. Trends Ecol. Evol. 28, 712-718. doi: 10.1016/j.tree.2013.09.013

Baumgartner, T., Götte, L., Gügler, R., and Fehr, E. (2012). The mentalizing network orchestrates the impact of parochial altruism on social norm enforcement. Hum. Brain Mapp. 33, 1452-1469. doi: 10.1002/hbm.21298
Finally, through language, spontaneous behavioral predispositions themselves can secondarily become the content of a norm, via representational redescription of pre-existing behavioral tendencies (Karmiloff-Smith, 1992). Above, we have exemplified this for the case of incest avoidance, a behavioral predisposition present in animals that in humans also takes the form of a cultural taboo (Turner and Maryanski, 2015). However, representational redescription also occurs in the case of the behavioral mechanisms of morality per se, such as prosocial concern. In this case, our prosocial concern may appear the result of purely cultural processes linked to language, whereas prosociality tests with primates have shown that it is more likely the simple convergent result of cooperative breeding, a general pattern that applies broadly.

In sum, we find that human morality had an important adaptive function in our hunter-gatherer ancestors in that it undergirded the extraordinarily interdependent lifestyle. Nevertheless, human morality was not invented from scratch in our hominin ancestors but could build on a rich set of pre-adaptations. A critical transition was the transformation of these elements to appear not only in individualistic and second-party contexts, but also in true third-party constellations. Based on evidence from the primate behavior and cognition literature, we are just beginning to understand how this transition and thus normativity emerged. Many new hypotheses emerge from this endeavor and will need to be tested in the future.

\section{AUTHOR CONTRIBUTIONS}

All authors listed have made a substantial, direct and intellectual contribution to the work, and approved it for publication.

\section{ACKNOWLEDGMENTS}

This research was supported by SNF grants 105312-114107 and 310030_130383 to JB and the Janggen Poehn Stiftung to RB.

Bierhoff, H.-W, and Rohmann, E. (2017). "Diffusion von Verantwortung," in Handbuch Verantwortung, eds L. Heidbrink, C Langbehn, and J Loh (Berlin: Springer), 911-931.

Bischof, N. (1975). "Comparative ethology of incest avoidance," in Biosocial Anthropology, ed R. Fox (London: Malaby Press), 37-67.

Boehm, C. (2012). Moral Origins: The Evolution of Virtue, Altruism, and Shame. New York, NY: Basic Books.

Brosnan, S. F., Talbot, C., Ahlgren, M., Lambeth, S. P., and Schapiro, S.J. (2010). Mechanisms underlying responses to inequitable outcomes in chimpanzees, Pan troglodytes. Anim. Behav. 79, 1229-1237. doi: 10.1016/j.anbehav.2010.02.019

Brügger, R. K., Kappeler-Schmalzriedt, T., and Burkart, J.M. (2018). Reverse audience effects on helping in cooperatively breeding common marmosets. Biol. Lett. 14:20180030. doi: 10.1098/rsbl.2018. 0030

Burkart, J. M., Allon, O., Amici, F., Fichtel, C., Finkenwirth, C., Heschl, A., et al. (2014). The evolutionary origin of human hyper-cooperation. Nat. Commun. 5:4747. doi: 10.1038/ncomms5747

Burkart, J. M., Fehr, E., Efferson, C., and van Schaik, C. P. (2007). Otherregarding preferences in a non-human primate: common marmosets 
provision food altruistically. Proc. Natl. Acad. Sci. U.S.A. 104, 19762-19766. doi: 10.1073/pnas.0710310104

Burkart, J. M., Hrdy, S. B., and van Schaik, C. P. (2009). Cooperative breeding and human cognitive evolution. Evol. Anthropol. 18, 175-186. doi: $10.1002 /$ evan.20222

Burkart, J. M., and Rueth, K. (2013). Preschool children fail primate prosocial game because of attentional task demands. PLOS ONE 8:e68440. doi: 10.1371/journal.pone.0068440

Burkart, J. M., Schubiger, M. N., and van Schaik, C. P. (2017a). The evolution of general intelligence. Behav. Brain Sci. 40, 1-65. doi: 10.1017/S0140525X16000959

Burkart, J. M., van Schaik, C., and Griesser, M. (2017b). Looking for unity in diversity: human cooperative childcare in comparative perspective. Proc. Biol. Sci. 284:20171184. doi: 10.1098/rspb.2017.1184

Burkart, J. M., and van Schaik, C. P. (2016). Revisiting the consequences of cooperative breeding. J. Zool. 299, 77-83. doi: 10.1111/jzo.12322

Capitanio, J. P., Blozis, S. A., Snarr, J., Steward, A., and McCowan, B. J. (2017). Do "birds of a feather flock together" or do "opposites attract"? Behavioral responses and temperament predict success in pairings of rhesus monkeys in a laboratory setting. Am. J. Primatol. 79, 1-11. doi: 10.1002/ajp.22464

Chudek, M., and Henrich, J. (2011). Culture-gene coevolution, norm-psychology and the emergence of human prosociality. Trends Cogn. Sci. 15, 218-226. doi: 10.1016/j.tics.2011.03.003

Clay, Z., Ravaux, L., de Waal, F. B., and Zuberbühler, K. (2016). Bonobos (Pan paniscus) vocally protest against violations of social expectations. J. Comp. Psychol. 130, 44-54. doi: 10.1037/a0040088

Cohen, E., Atkinson, Q. D., Dediu, D., Dingemanse, M., Kinzler, K., Ladd, D.R., et al. (2012). The evolution of tag-based cooperation in humans: the case for accent. Curr. Anthropol. 53, 588-616. doi: 10.1086/667654

Cronin, K. A. (2012). Prosocial behaviour in animals: the influence of social relationships, communication and rewards. Anim. Behav. 84, 1085-1093. doi: 10.1016/j.anbehav.2012.08.009

Dean, L. G., Vale, G. L., Laland, K. N., Flynn, E., and Kendal, R. L. (2014). Human cumulative culture: a comparative perspective. Biol. Rev. 89, 284-301. doi: 10.1111/brv.12053

Decety, J., and Yoder, K.J. (2017). The emerging social neuroscience of justice motivation. Trends Cogn. Sci. 21, 6-14. doi: 10.1016/j.tics.2016.10.008

Dreu, C. K., Dussel, D. B., and Velden, F. S. (2015). In intergroup conflict, self-sacrifice is stronger among pro-social individuals, and parochial altruism emerges especially among cognitively taxed individuals. Front. Psychol. 6:572. doi: $10.3389 /$ fpsyg.2015.00572

Delton, A. W., and Krasnow, M. M. (2017). The psychology of deterrence explains why group membership matters for third-party punishment. Evol. Hum. Behav. 38, 734-743. doi: 10.1016/j.evolhumbehav.2017.07.003

DeScioli, P., and Kurzban, R. (2013). A solution to the mysteries of morality. Psychol. Bull. 139, 477-496. doi: 10.1037/a0029065

de Waal, F. B. M. (1991). The chimpanzee's sense of social regularity and its relation to the human sense of justice. Am. Behav. Sci. 34, 335-349. doi: 10.1177/0002764291034003005

de Waal, F. B. M. (2006). Primates and Philosophers. Princeton, NJ: Princeton University Press.

Dindo, M., Whiten, A., and de Waal, F. B. (2009). In-group conformity sustains different foraging traditions in capuchin monkeys (Cebus apella). PLoS ONE 4:e7858. doi: 10.1371/journal.pone.0007858

Engelmann, J. M., Clift, J. B., Herrmann, E., and Tomasello, M. (2017). Social disappointment explains chimpanzees' behaviour in the inequity aversion task. Proc. R. Soc. B 284:20171502. doi: 10.1098/rspb.2017.1502

Engelmann, J. M., Herrmann, E., and Tomasello, M. (2012). Five-year olds, but not chimpanzees, attempt to manage their reputations. PLoS ONE 7:e48433. doi: 10.1371/journal.pone.0048433

Fehr, E., and Fischbacher, U. (2003). The nature of human altruism. Nature 423, 785-791. doi: 10.1038/nature02043

Fessler, D. M. T., Barrett, H. C., Kanovsky, M., Stich, S., Holbrook, C., Henrich, J., et al. (2015). Moral parochialism and contextual contingency across seven societies. Proc. R. Soc. B 282:20150907. doi: 10.1098/rspb.2015.0907

Fessler, D. M. T., and Navarrete, C. D. (2004). Third-party attitudes toward sibling incest: evidence for Westermarck's hypotheses. Evol. Hum. Behav. 25, 277-294. doi: 10.1016/j.evolhumbehav.2004.05.004
Gelfand, M. J., and Jackson, J. C. (2016). From one mind to many: the emerging science of cultural norms. Curr. Opin. Psychol. 8, 175-181. doi: 10.1016/j.copsyc.2015.11.002

Goffman, E. (1959). The Presentation of Self in Everyday Life. Garden City, NY: Doubleday/Anchor.

Goodall, J. (1971). In the Shadow of Man. Boston, MA: Houghton Mifflin Harcourt.

Goodall, J. (1977). Infant killing and cannibalism in free-living chimpanzees. Folia Primatol. 28, 259-282. doi: 10.1159/000155817

Gruber, T., Zuberbühler, K., Clément, F., and van Schaik, C. (2015). Apes have culture but may not know that they do. Front. Psychol. 6:91. doi: 10.3389/fpsyg.2015.00091

Guala, F. (2012). Reciprocity: weak or strong? What punishment experiments do (and do not) demonstrate. Behav. Brain Sci. 35, 1-15. doi: 10.1017/S0140525X11000069

Gurven, M., Allen-Arave, W., Hill, K., and Hurtado, M. (2000). "It's a wonderful life": signaling generosity among the Ache of Paraguay. Evol. Hum. Behav. 21, 263-282. doi: 10.1016/S1090-5138(00)00032-5

Haidt, J. (2013). Moral psychology for the twenty-first century. J. Moral Educ. 42, 281-297. doi: 10.1080/03057240.2013.817327

Hamlin, J. K., Wynn, K., and Bloom, P. (2007). Social evaluation by preverbal infants. Nature 450, 557-559. doi: 10.1038/nature06288

Hare, R. D. (1999). Without Conscience: The Disturbing World of the Psychopaths Among Us. New York, NY: Guilford Press.

Haun, D. B. M., and Over, H. (2015). "Like me: a homophily-based account of human culture," in Cultural Evolution: Society, Technology, Language, and Religion, eds P. J. Richerson and M. H. Christiansen (Cambridge, MA: MIT Press), 75-85.

Hector, A. C., Seyfarth, R. M., and Raleigh, M. J. (1989). Male parental care, female choice, and the effect of an audience in vervet monkeys. Anim. Behav. 38, 262-271. doi: 10.1016/S0003-3472(89)80088-0

Henrich, J., and Broesch, J. (2011). On the nature of cultural transmission networks: evidence from Fijian villages for adaptive learning biases. Philos. Trans. R. Soc. Lond. B Biol. Sci. 366, 1139-1148. doi: 10.1098/rstb.2010.0323

Hill, K., Walker, R. S., BoŽičević, M., Eder, J., Headland, T., Hewlett, B. S., et al. (2011). Co-residence patterns in hunter-gatherer societies show unique human social structure. Science 331, 1286-1289. doi: 10.1126/science.1199071

Hill, K., Wood, B. M., Baggio, J., Hurtado, A. M., and Boyd, R. T. (2014). Huntergatherer inter-band interaction rates: implications for cumulative culture. PLoS ONE 9:e102806. doi: 10.1371/journal.pone.0102806

Hopper, L. M., Schapiro, S. J., Lambeth, S. P., and Brosnan, S. F. (2011). Chimpanzees' socially maintained food preferences indicate both conservatism and conformity. Anim. Behav. 81, 1195-1202. doi: 10.1016/j.anbehav.2011.03.002

Hrdy, S. (2009). Mothers \& Others: The Evolutionary Origins of Mutual Understanding. Cambridge: Harvard University Press.

Hrubesch, C., Preuschoft, S., and van Schaik, C.P. (2009). Skill mastery inhibits adoption of observed alternative solutions among chimpanzees (Pan troglodytes). Anim. Cogn. 12, 209-216. doi: 10.1007/s10071-008-0183-y

Isler, K., and Van Schaik, C. P. (2012). How our ancestors broke through the gray ceiling: comparative evidence for cooperative breeding in early homo. Curr. Anthropol. 53, S453-S465. doi: 10.1086/667623

Jaeggi, A., Burkart, J. M., and van Schaik, C. P. (2010). On the psychology of cooperation in humans and other primates: the natural history of food sharing and experimental evidence of prosociality. Philos. Trans. R. Soc. B Biol. Sci. 12, 2723-2735. doi: 10.1098/rstb.2010.0118

Kanngiesser, P., and Warneken, F. (2012). Young children consider merit when sharing resources with others. PLoS ONE 7:e43979. doi: 10.1371/journal.pone.0043979

Kaplan, H. S., Hooper, P. L., and Gurven, M. (2009). The evolutionary and ecological roots of human social organization. Philos. Trans. R. Soc. B Biol. Sci. 364, 3289-3299. doi: 10.1098/rstb.2009.0115

Karmiloff-Smith, A. (1992). Beyond Modularity: A Developmental Approach to Cognitive Science. Cambridge, MA: MIT Press.

Krasnow, M. M., Cosmides, L., Pedersen, E. J., and Tooby, J. (2012). What are punishment and reputation for? PLOS ONE 7:e45662. doi: 10.1371/journal.pone.0045662

Krupenye, C., and Hare, B. (2018). Bonobos prefer individuals that hinder others over those that help. Curr. Biol. 28, 280-286.e5. doi: 10.1016/j.cub.2017.11.061 
Krupenye, C., Kano, F., Hirata, S., Call, J., and Tomasello, M. (2016). Great apes anticipate that other individuals will act according to false beliefs. Science 354, 110-114. doi: 10.1126/science.aaf8110

Kuzawa, C. W., Chugani, H. T., Grossman, L. I., Lipovich, L., Muzik, O., Hof, P. R., et al. (2014). Metabolic costs and evolutionary implications of human brain development. Proc. Natl. Acad. Sci. U.S.A. 111, 13010-13015. doi: 10.1073/pnas.1323099111

Latané, B., and Darley, J. M. (1969). Bystander "Apathy". Am. Sci. 57, 244-268.

Long, A.A., and Sedley, D.N. (1987). The Hellenistic Philosophers. I: Translations of the Principal Sources with Philosophical Commentary; II: Greek and Latin Texts with Notes and Bibliography. Cambridge: Cambridge University Press.

Luncz, L.V., and Boesch, C. (2014). Tradition over trend: neighboring chimpanzee communities maintain differences in cultural behavior despite frequent immigration of adult females. Am. J. Primatol. 76, 649-657. doi: 10.1002/ajp.22259

MacLean, E.L. (2016). Unraveling the evolution of uniquely human cognition. Proc. Natl. Acad. Sci. U.S.A. 113, 6348-6354. doi: 10.1073/pnas.1521270113

Marlowe, F. (2005). Hunter-gatherers and human evolution. Evol. Anthropol. 14, 54-67. doi: 10.1002/evan.20046

Marlowe, F. (2007). Hunting and gathering: the human sexual division of foraging labor. Cross Cult. Res. 41, 170-195. doi: 10.1177/1069397106297529

Marlowe, F. (2010). The Hadza: Hunter-gatherers of Tanzania. Berkeley, CA: University of California Press.

Marshall-Pescini, S., Dale, R., Quervel-Chaumette, M., and Range, F. (2016). Critical issues in experimental studies of prosociality in non-human species. Anim. Cogn. 19, 1-27. doi: 10.1007/s10071-016-0973-6

Massen, J. J. M., and Koski, S. E. (2014). Chimps of a feather sit together: chimpanzee friendships are based on homophily in personality. Evol. Hum. Behav. 35, 1-8. doi: 10.1016/j.evolhumbehav.2013. 08.008

Migliano, A. B., Page, A. E., Gómez-Gardeñes, J., Salali, G. D., Viguier, S., Dyble, M., et al. (2017). Characterization of hunter-gatherer networks and implications for cumulative culture. Nat. Hum. Behav. 1:0043. doi: 10.1038/s41562-016-0043

Nettle, D., Cronin, K., and Bateson, M. (2013a). Responses of chimpanzees to cues of conspecific observation. Anim. Behav. 86, 595-602. doi: 10.1016/j.anbehav.2013.06.015

Nettle, D., Harper, Z., Kidson, A., Stone, R., Penton-Voak, I.S., and Bateson, M. (2013b). The watching eyes effect in the Dictator Game: it's not how much you give, it's being seen to give something. Evol. Hum. Behav. 34, 35-40. doi: 10.1016/j.evolhumbehav.2012.08.004

Paukner, A., Suomi, S. J., Visalberghi, E., and Ferrari, P.F. (2009). Capuchin monkeys display affiliation toward humans who imitate them. Science 325, 880-883. doi: 10.1126/science.1176269

Paulus, M. (2014). The early origins of human charity: developmental changes in preschoolers' sharing with poor and wealthy individuals. Front. Psychol. 5:344. doi: 10.3389/fpsyg.2014.00344

Piazza, J., and Sousa, P. (2016). When injustice is at stake, moral judgements are not parochial. Proc. R. Soc. B 283:20152037. doi: 10.1098/rspb.2015.2037

Pusey, A., and Wolf, M. (1996). Inbreeding avoidance in animals. Trends Ecol. Evol. 11, 201-206. doi: 10.1016/0169-5347(96)10028-8

Ruch, H., Zürcher, Y., and Burkart, J. M. (2018). The function of vocal accommodation in humans and other primates. Biol. Rev. 92, 996-1013. doi: 10.1111/brv.12382

Rudolf von Rohr, C., Burkart, J. M., and van Schaik, C. P. (2011). Evolutionary precursors of social norms in chimpanzees: a new approach. Biol. Philos. 26, 1-30. doi: 10.1007/s10539-010-9240-4

Rudolf von Rohr, C., Koski, S. E., Burkart, J. M., Caws, C., Fraser, O. N., Ziltener, A., et al. (2012). Impartial third-party interventions in captive chimpanzees: a reflection of community concern. PLoS ONE 7:e32494. doi: 10.1371/journal.pone.0032494

Rudolf von Rohr, C., van Schaik, C. P., Kissling, A., and Burkart, J. M. (2015). Chimpanzees' bystander reactions to infanticide. Hum. Nat. 26, 143-160. doi: 10.1007/s12110-015-9228-5

Saltzman W. (2003). "Reproductive competition among female common marmosets (Callithrix jacchus): proximate and ultimate causes," in Sexual Selection and Reproductive Competition in Primates, ed C. B. Jones (Norman, OK: American Society of Primatologists), 197-229.
Saltzman, W., Pick, R. R., Salper, O. J., Liedl, K. J., and Abbott, D. H. (2004). Onset of plural cooperative breeding in common marmoset families following replacement of the breeding male. Anim. Behav. 68, 59-73. doi: 10.1016/j.anbehav.2003.07.020

Sear, R., and Mace, R. (2008). Who keeps children alive? A review of the effects of kin on child survival. Evol. Hum. Behav. 29, 1-18. doi: 10.1016/j.evolhumbehav.2007.10.001

Sugiyama, L. S., and Chacon, R. (2005). Juvenile Responses to Household Ecology Among the Yora of Peruvian Amazonia. Hunter-Gatherer Childhoods: Evolutionary, Developmental and Cultural Perspectives. New Brunswick, NJ: Aldine Transaction, 237-261.

Talbot, C.F., Price, S. A., and Brosnan, S. F. (2016). "Inequity responses in nonhuman animals," in Handbook of Social Justice Theory and Research (New York, NY: Springer), 387-403.

Tan, J., Ariely, D., and Hare, B. (2017). Bonobos respond prosocially toward members of other groups. Sci. Rep. 7:14733. doi: 10.1038/s41598-017-15320-w

Tan, J., and Hare, B. (2013). Bonobos Share with Strangers. PLoS ONE 8:e51922. doi: 10.1371/journal.pone.0051922

Tan, J., Kwetuenda, S., and Hare, B. (2015). Preference or paradigm? Bonobos show no evidence of other-regard in the standard prosocial choice task. Behaviour 152, 521-544. doi: 10.1163/1568539X-00003230

Tennie, C., Jensen, K., and Call, J. (2016). The nature of prosociality in chimpanzees. Nat. Commun. 7:13915. doi: 10.1038/ncomms13915

Tinbergen, N. (1963). On aims and methods of ethology. Zeitschr. Tierpsychol. 20, 410-433. doi: 10.1111/j.1439-0310.1963.tb01161.x

Tomasello, M. (2016). A Natural History of Human Morality. Harvard, MA: Harvard University Press.

Tomasello, M., and Gonzalez-Cabrera, I. (2017). The role of ontogeny in the evolution of human cooperation. Hum. Nat. 28, 274-288. doi: 10.1007/s12110-017-9291-1

Tomasello, M., Melis, A. P., Tennie, C., Wyman, E., and Herrmann, E. (2012). Two key steps in the evolution of human cooperation. Curr. Anthropol. 53, 673-692. doi: $10.1086 / 668207$

Townsend, S. W., Slocombe, K. E., Thompson, M. E., and Zuberbühler, K. (2007). Female-led infanticide in wild chimpanzees. Curr. Biol. 17, R355-R356. doi: 10.1016/j.cub.2007.03.020

Turner, J. H., and Maryanski, E. (2015). Incest: Origins of the Taboo. New York, NY: Routledge.

Ueno, A., and Matsuzawa, T. (2004). Food transfer between chimpanzee mothers and their infants. Primates 45, 231-239. doi: 10.1007/s10329-004-0 085-9

Ulber, J., Hamann, K., and Tomasello, M. (2017). Young children, but not chimpanzees, are averse to disadvantageous and advantageous inequities. J. Exp. Child Psychol. 155, 48-66. doi: 10.1016/j.jecp.2016. 10.013

van de Waal, E., Borgeaud, C., and Whiten, A. (2013). Potent social learning and conformity shape a wild primate's foraging decisions. Science 340, 483-485. doi: 10.1126/science. 1232769

van de Waal, E., van Schaik, C. P., and Whiten, A. (2017). Resilience of experimentally seeded dietary traditions in wild vervets: evidence from group fissions. Am. J. Primatol. 79:e22687. doi: 10.1002/ajp. 22687

Van Leeuwen, E. J. C., Kendal, R. L., Tennie, C., and Haun, D. B. M. (2015). Conformity and its look-a-likes. Anim. Behav. 110, e1-e4. doi: 10.1016/j.anbehav.2015.07.030

van Schaik, C. P. (2016). The Primate Origins of Human Nature. New York, NY: John Wiley \& Sons.

van Schaik, C. P. M. A., Djojoasmoro, R., Knott, C. D., and Morrogh-Bernard, H.C.(eds.) (2009). "Orangutan cultures revisited," in Orangutans: Georgraphic Variation in Behavioral Ecology and Conservation (New York, NY: Oxford University Press), 299-309.

van Schaik, C. P., and Burkart, J. M. (2010). "Mind the gap: cooperative breeding and the evolution of our unique features," in Mind the Gap: Tracing the Origins of Human Universals, eds P. M. Kappeler and J. Silk. (Heidelberg: Springer), 477-496.

van Schaik, C. P., Burkart, J. M., Jaeggi, A., and Rudolf von Rohr, C. (2014). "Morality as biological adaptation-an evolutionary model based on the lifestyle of human foragers," in Empirically Informed Ethics: Morality between Facts and 
Norms, eds M. Christen, J. Fischer, M. Huppenbauer, C. Tanner, and C. van Schaik (Cham: Springer International Publishing), 65-84.

van Schaik, C. P., and Janson, C. H. (2000). Infanticide by Males and Its Implications. Cambridge: Cambridge University Press.

Warneken, F., and Tomasello, M. (2015). "The developmental and evolutionary origins of human helping and sharing," in Oxford Handbooks Online, eds D. A. Schroeder and W. G. Graziano (Oxford, UK: Oxford University Press), 100-113. doi: 10.1093/oxfordhb/9780195399813.013.007

Weaver, G. R., Reynolds, S. J., and Brown, M. E. (2014). Moral intuition: connecting current knowledge to future organizational research and practice. J. Manag. 40, 100-129. doi: 10.1177/0149206313511272

Whiten, A., Goodall, J., McGrew, W. C., Nishida, T., Reynolds, V., Sugiyama, Y., et al. (2017). "Cultures in chimpanzees," in The Animal Ethics Reader, eds S. J. Armstrong and R. G. Botzler (New York, NY: Routledge), 182-184.

Whiten, A., Horner, V., and de Waal, F. B. M. (2005). Conformity to cultural norms of tool use in chimpanzees. Nature 437, 737-740. doi: 10.1038/nature04047

Whiten, A., Spiteri, A., Horner, V., Bonnie, K. E., Schapiro, S. J., and de Waal, F. B. M. (2007). Transmission of multiple traditions within and between chimpanzee groups. Curr. Biol. 17, 1-6. doi: 10.1016/j.cub.2007. 05.031

Whiten, A., and van de Waal, E. (2016a). Identifying and dissecting conformity in animals in the wild: further analysis of primate data. Anim. Behav. 122, e1-e4. doi: 10.1016/j.anbehav.2016.04.002
Whiten, A., and van de Waal, E. (2016b). Social learning, culture and the 'sociocultural brain'of human and non-human primates. Neurosci. Biobehav. Rev. 82, 58-75. doi: 10.1016/j.neubiorev.2016.12.018

Wood, B. M., and Marlowe, F. W. (2013). Household and kin provisioning by Hadza men. Hum. Nat. 24, 280-317. doi: 10.1007/s12110-013-9173-0

Wrangham, R. (2009). Catching Fire: How Cooking Made us Human. New York, NY: Basic Books.

Wrangham, R. W., Koops, K., Machanda, Z. P., Worthington, S., Bernard, A. B., Brazeau, N. F., et al. (2016). Distribution of a chimpanzee social custom is explained by matrilineal relationship rather than conformity. Curr. Biol. 26, 3033-3037. doi: 10.1016/j.cub.2016.09.005

Conflict of Interest Statement: The authors declare that the research was conducted in the absence of any commercial or financial relationships that could be construed as a potential conflict of interest.

Copyright (c) 2018 Burkart, Brügger and van Schaik. This is an open-access article distributed under the terms of the Creative Commons Attribution License (CC BY). The use, distribution or reproduction in other forums is permitted, provided the original author(s) and the copyright owner(s) are credited and that the original publication in this journal is cited, in accordance with accepted academic practice. No use, distribution or reproduction is permitted which does not comply with these terms. 